\title{
US hereditary ataxia mutation frequencies compared to EFNS molecular testing guidelines
}

\author{
Zhenyuan Wang ${ }^{1}$, Christopher M Gomez ${ }^{2}$, David A Hill ${ }^{3}$, Marc A Meservey ${ }^{4}$, Michele McCarthy ${ }^{5}$, Sat Dev Batish ${ }^{6}$ and Joseph J Higgins $^{7 *}$ \\ ${ }^{1}$ Zhenyuan Wang, Quest Diagnostics, Marlborough, MA 01752 USA \\ ${ }^{2}$ Christopher M Gomez, Department of Neurology, University of Chicago, Chicago, IL 60637 USA \\ ${ }^{3}$ David A Hill, Quest Diagnostics, Marlborough, MA 01752 USA \\ ${ }^{4}$ Marc A Meservey, Quest Diagnostics, Marlborough, MA 01752 USA \\ ${ }^{5}$ Michele McCarthy, Quest Diagnostics, Marlborough, MA 01752 USA \\ ${ }^{6}$ Sat Dev Batish, Quest Diagnostics, Marlborough, MA 01752 USA \\ 7Joseph J Higgins, Quest Diagnostics, Marlborough, MA 01752 USA
}

\begin{abstract}
Objective: To describe the mutation frequencies in ataxia genes referred to a clinical laboratory for genetic testing and compare the data with the 2014 European Federation of Neurological Societies (EFNS) testing guidelines.

Methods: Specimens ( $\mathrm{n}=3,394)$ submitted for hereditary ataxia testing included a 20-gene comprehensive profile ( $\mathrm{n}=2,165)$, a 14 -gene autosomal dominant (AD) profile ( $\mathrm{n}=812)$, or a 6-gene autosomal recessive (AR) profile ( $\mathrm{n}=417)$. The genes associated with AD inheritance included $A T N 1$ (DRPLA), $A T X N 1$ (SCA1), ATXN2 (SCA2), ATXN3 (SCA3), SPTBN2 (SCA5), CACNA1A (SCA6), ATXN7 (SCA7), ATXN8OS (SCA8), ATXN10 (SCA10), PPP2R2B (SCA12), KCNC3 (SCA13), PRKCG (SCA14), TBP (SCA17), and AFG3L2 (SCA28). The genes associated with AR inheritance included $A P T X, F X N$ [Friedreich ataxia (FRDA)], POLG, SETX, SIL1, and TTPA. Repeat expansions were analyzed by PCR, repeat-primed PCR, and Southern blot assay. Sequence variants were analyzed by Sanger sequencing.

Results: The comprehensive profile yielded positive results for one or more genes in 254 (11.7\%) specimens including 204 (80.3\%) AD, and 50 (19.7\%) AR. Most positive findings (92.9\%) were repeat expansions with 7.1\% pathogenic variants. Expansions in SCA8 (18.1\%) were the most common positive results followed by SCA3 and SCA6 (16.1\% each), FRDA (15.7\%), SCA2 (13.8\%), SCA1 (6.7\%), SCA10 (2.4\%), SCA17 (2.0\%), SCA7 (1.6\%), and SCA12 (0.4\%). SCA1, 2, 3, 6, 7 , and 17 accounted for $81.6 \%$ of positive results in the AD profile while FRDA accounted for $78.9 \%$ in the AR profile.
\end{abstract}

Conclusion: The mutation frequencies reported, except for SCA8 and SCA10, are consistent with the EFNS genetic testing recommendations for ataxia.

\section{Introduction}

The hereditary ataxias are characterized by progressive incoordination that mainly affects ambulation but often involves the upper extremities, speech, and eye movements [1]. Prevalence varies across populations with estimates ranging from 1 to 9 per 100,000 people [2-5]. Inheritance patterns can be autosomal dominant (AD), autosomal recessive (AR), X-linked, or maternal if part of a mitochondrial genetic syndrome [1]. The most frequent inheritance pattern is $\mathrm{AD}$, which is generally associated with an adult onset. The most common spinocerebellar ataxia (SCA) loci 1, 2, 3, 6, and 7 are characterized by CAG trinucleotide repeat expansions in their respective genes [1]. Among the AR ataxias, which usually have a childhood onset, Friedreich ataxia (FRDA) is the most common with a disease frequency of 1:20,000 to 1:50,000 [1].

Diagnosing specific ataxia subtypes has important treatment and prognostic implications. The diagnosis typically requires ataxia, a detailed family history, the identification of additional clinical features, and neuroimaging. Molecular testing plays a prominent diagnostic role because of the difficulty differentiating subtypes based on clinical grounds alone [1]. In 2014, the European Federation of Neurological Societies (EFNS) recommended initial genetic testing for $\mathrm{AD}$ ataxia, including SCA1, 2, 3, 6, 7, and 17 and one gene, FXN (FRDA); it recommended testing for $\mathrm{AR}$ ataxia when cerebellar atrophy is absent on brain MRI $[6,7]$. These seven loci (SCA1, 2, 3, $6,7,17$, and FRDA) represent the majority of hereditary ataxias in Europe and more than $60 \%$ of AD cases worldwide [8]. The EFNS did not include SCA10 or dentatorubral-pallidoluysian atrophy (DRPLA) in their recommendations because pathogenic variants in these genes are restricted to patients with ancestral ties to Latin American or Asian countries $[6,7,9]$. Although SCA 8 accounts for $2 \%$ to $5 \%$ of AD ataxia,

Correspondence to: Joseph J. Higgins, MD, FAAN, Quest Diagnostics, 200 Forest Street, 2nd Floor, Marlborough, MA 01752 USA, Tel: 774.843.3246, Fax: 508.659.0820, E-mail: Joseph.J.Higgins@QuestDiagnostics.com

Key words: Hereditary ataxia, spinocerebellar ataxia, Friedreich ataxia, mutation, genetic testing, autosomal dominant, autosomal recessive

Received: August 20,2017; Accepted: September 13, 2017; Published: September 15,2017 
the EFNS did not recommend testing for this subtype in initial screening because the causative expanded allele has a low penetrance $[6,7,10]$.

The EFNS recommendations for molecular diagnosis of ataxia are designed to assist European neurologists in using genetic testing to diagnose specific ataxia subtypes [6,7]. To evaluate the EFNS recommendations for testing specific ataxia genes, we retrospectively assessed the frequencies and types of mutations in specimens referred to a clinical laboratory in the USA.

\section{Methods}

This study used de-identified laboratory results from consecutive specimens submitted for ataxia genetic testing between 2011 and 2014. Less than $1 \%$ of specimens were received from outside the USA. The results from sequencing and repeat expansion assays were extracted from an internal database by gene name without identifying information. In all cases, healthcare providers ordered genetic testing on a standardized requisition form and provided the clinical indications and ICD codes. Detailed clinical information and the relatedness between patients were unavailable.

The study included 3,394 patient specimens, 2,165 of which were tested using a comprehensive profile of 20 genes: 14 associated with $\mathrm{AD}$ and 6 associated with AR ataxias (Table 1). The remaining specimens were tested with a 14 -gene AD profile $(n=812)$ or a 6-gene AR profile $(n=417)$ (Table 1). Non-repeat expansions were detected by DNA sequencing, which was performed by PCR amplification of purified genomic DNA, followed by Sanger DNA sequencing of the coding regions of all the genes except POLG and KCNC3. POLG gene exons 7,13 , and 21 and $K C N C 3$ gene exon 2 were sequenced and analyzed. Abnormal sequence variants were confirmed using bi-directional sequencing. Repeat expansions were detected by PCR amplification of the repeat region followed by high-resolution electrophoresis to determine the number of tandem repeats in each allele. Repeat primed assay or Southern blot analysis was used to confirm the homozygosity of normal alleles. A two-tailed Fisher's exact test was used to compare the differences in the positive rates between the EFNS [7] and the clinical laboratory datasets.

\section{Results}

Of the 2,165 patients tested with the comprehensive profile, 254 (11.7\%) were positive for repeat expansions, pathogenic variants, or likely pathogenic variants: $204(80.3 \%)$ in $\mathrm{AD}$ and 50 (19.7\%) in AR ataxia genes. Repeat expansions accounted for 92.9\% (236/254) of the positive results, and pathogenic or likely pathogenic variants for only $7.1 \%(18 / 254)$. The positive rates by gene for the comprehensive profiles are shown in Figure 1A. Repeat expansions in genes associated with $\mathrm{AD}$ ataxia collectively accounted for $196(77.2 \%)$ of the 254 positive results. SCA8 expansions were the most frequent accounting for $18.1 \%$ of the 254 positive results, followed by SCA3 (16.1\%), SCA6 (16.1\%), SCA2 (13.8\%), SCA1 (6.7\%), SCA10 (2.4\%), SCA17 (2.0\%), SCA7 (1.6\%), and SCA12 (0.4\%); no DRPLA mutations were detected (Figure 1A). There were no differences in the regional geographic distribution of positive results, except for SCA10 $(n=24)$ : positive results clustered in California (14/24) and Texas (3/24). Repeat expansions in FXN (FRDA) accounted for $15.7 \%$ of positive results (Figure 1A). The positive rates for both the $\mathrm{AD}$ profile $(20.1 \%, 163 / 812)$ (Figure $1 \mathrm{~B})$ and AR profile $(4.6 \%, 19 / 417)$ (Figure 1C) were higher than those for genes within the comprehensive profile. For the comprehensive profile, the positive rates were $9.4 \%(204 / 2,165)$ for genes associated with AD and $2.3 \%(50 / 2,165)$ for genes associated with AR ataxia. The $6 \mathrm{AD}$ genes recommended by the EFNS, ATXN1 (SCA1), ATXN2 (SCA2), ATXN3 (SCA3), CACNA1A (SCA6), ATXN7 (SCA7), and TBP (SCA17)
Table 1. Ataxia genes (loci) included in multigene profiles tested in a US clinical laboratory

\begin{tabular}{|c|c|c|}
\hline Comprehensive Profile & Dominant Profile & Recessive Profile \\
\hline ATN1 (DRPLA) & ATN1 (DRPLA) & $A P T X$ \\
\hline$A F G 3 L 2$ (SCA28) & $A F G 3 L 2$ (SCA28) & $F X N($ FRDA $)$ \\
\hline$A P T X$ & ATXN1 (SCA1) & $P O L G$ \\
\hline$A T X N 1$ (SCA1) & $A T X N 2$ (SCA2) & SETX \\
\hline$A T X N 2$ (SCA2) & ATXN3 (SCA3) & SIL1 \\
\hline$A T X N 3(\mathrm{SCA} 3)$ & ATXN7 (SCA7) & TTPA \\
\hline$A T X N 7$ (SCA7) & ATXN8OS (SCA8) & \\
\hline ATXN8OS (SCA8) & CACNAIA (SCA6) & \\
\hline CACNA1A (SCA6) & ATXN10 (SCA10) & \\
\hline ATXN10 (SCA10) & KCNC3 (SCA13) & \\
\hline$F X N($ FRDA $)$ & $P P P 2 R B 2$ (SCA12) & \\
\hline$K C N C 3$ (SCA13) & $P R K C G$ (SCA14) & \\
\hline$P O L G$ & SPTBN2 (SCA5) & \\
\hline$P P P 2 R B 2$ (SCA12) & $T B P(\mathrm{SCA} 17)$ & \\
\hline \multicolumn{3}{|l|}{$P R K C G$ (SCA14) } \\
\hline \multicolumn{3}{|l|}{ SETX } \\
\hline \multicolumn{3}{|l|}{ SILI } \\
\hline \multicolumn{3}{|l|}{ SPTBN2 (SCA5) } \\
\hline \multicolumn{3}{|l|}{ TBP (SCA17) } \\
\hline TTPA & & \\
\hline
\end{tabular}

accounted for $70.1 \%(n=143)$ of the positive results for $\mathrm{AD}$ ataxia genes $(n=204)$ in the comprehensive profile (Figure 2A). These frequencies were not significantly different $(\mathrm{P}=0.095)$ than the frequencies reported by the EFNS (61.9\%) [7]. However, these 6 genes accounted for $81.6 \%$ in the $\mathrm{AD}$-only profile which is significantly more than expected $(\mathrm{P}<0.001)$ compared to the EFNS data (Figure 2A) [7]. The observed frequencies of positive results for FRDA (80.0\%) in the comprehensive AR profile and in the AR-only profile (78.9\%) were not significantly $(\mathrm{P}=0.810)$ different from the EFNS frequency $(75.3 \%)$ reported for the European population (Figure 2B).

\section{Discussion}

The positive rates in this study may be overestimates due to the lack of detailed clinical information and the possibility that more than one family member was tested. Overall, the data agrees with the 2014 EFNS guidelines for genetic testing for ataxia with two exceptions $[6,7]$. The first exception is that SCA10 is not included in the EFNS recommendation because it has been only been reported in individuals from Latin American or of Native American descent [9]. However, in our study population, SCA10 accounted for $2.4 \%$ of our positive specimens; we noted a regional clustering in California and Texas likely due to founder effects. The second exception is that repeat expansions in SCA8 were the most common positive result (18.1\%) in the comprehensive profile. The high rate of co-occurrence with pathogenic variants in other $\mathrm{AD}$ ataxia genes in this study (10/46 specimens: 4 SCA8 expansions on both alleles, 4 with other repeat expansions, and 2 with a sequencing variant in $P O L G$ ) suggest that repeat expansions in SCA8 may not cause ataxia directly in some cases. In addition, not all SCA8 expansions are associated with progressive ataxia suggesting the co-occurrence is due to its low penetrance $[8,10]$. The role of SCA8 as a genetic modifier and the mechanisms for incomplete penetrance require further study.

The comprehensive profile detected $\mathrm{AD}$ mutations in nearly $10 \%$ of ataxia patients and AR mutations in only $2.3 \%$ in our heterogeneous sample. When an $\mathrm{AD}$ or $\mathrm{AR}$ profile was ordered, the frequency of positive results roughly doubled. This suggests that the AD-only or AR-only profiles should be performed first in patients with a known inheritance pattern. 
A

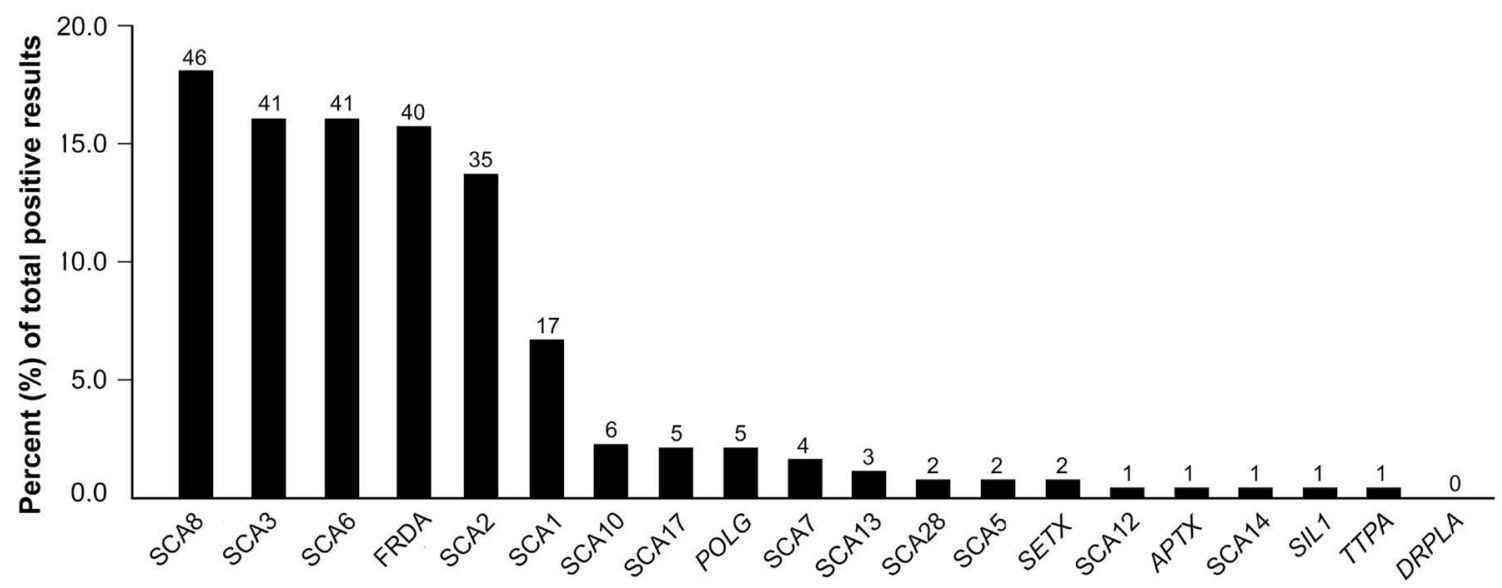

B

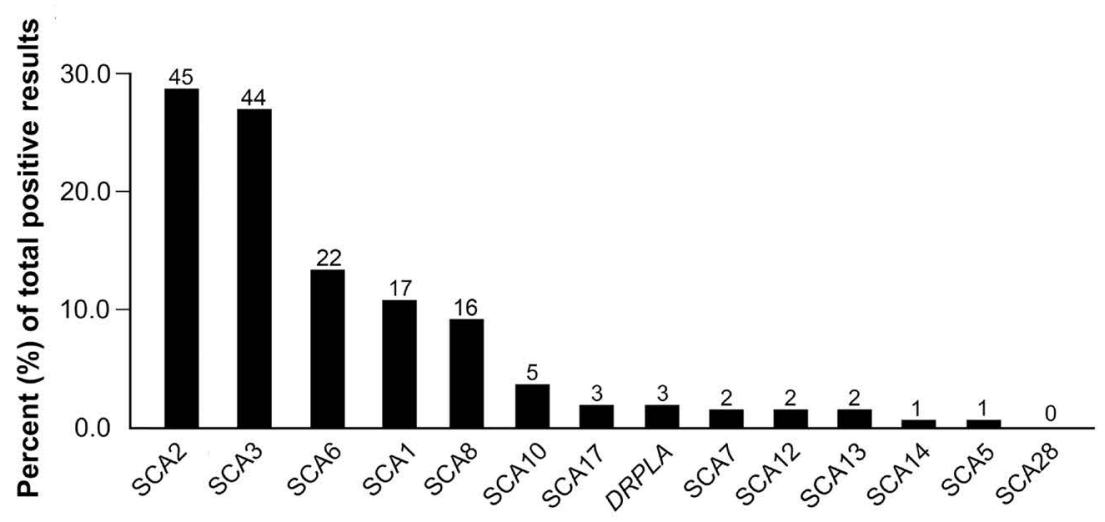

C

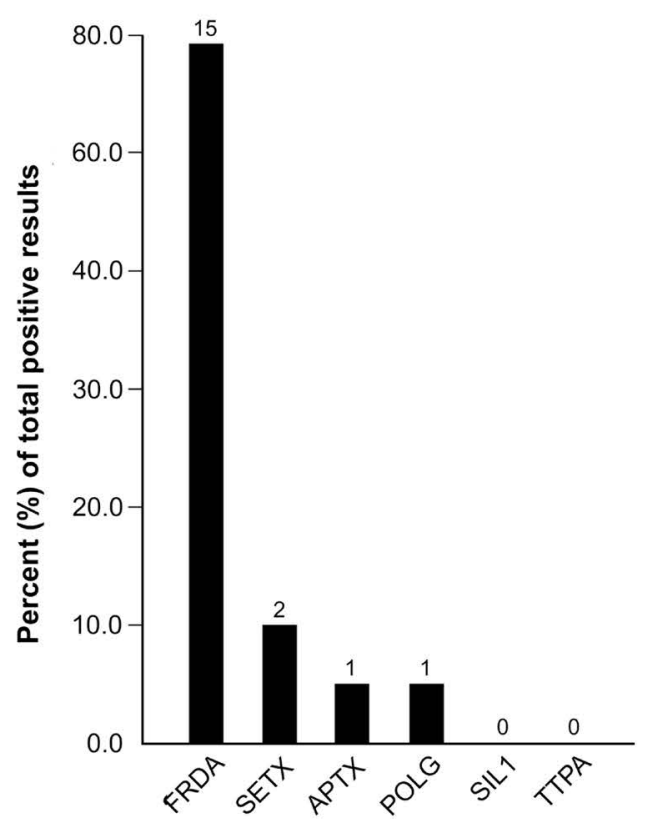

Figure 1. The frequency of positive test results for loci or genes in the comprehensive (A), AD (B), or AR (C) profiles ( $\mathrm{n}=436$ ). The percent of pathogenic or likely pathogenic test results for each profile are shown. The numbers above the black bars are the numbers of positive results in the total numbers of positive tests for the comprehensive ( $\mathrm{n}=254$ ), $\mathrm{AD}$ ( $\mathrm{n}=163$ ) and $\mathrm{AR}$ profiles $(\mathrm{n}=19)$. 
A

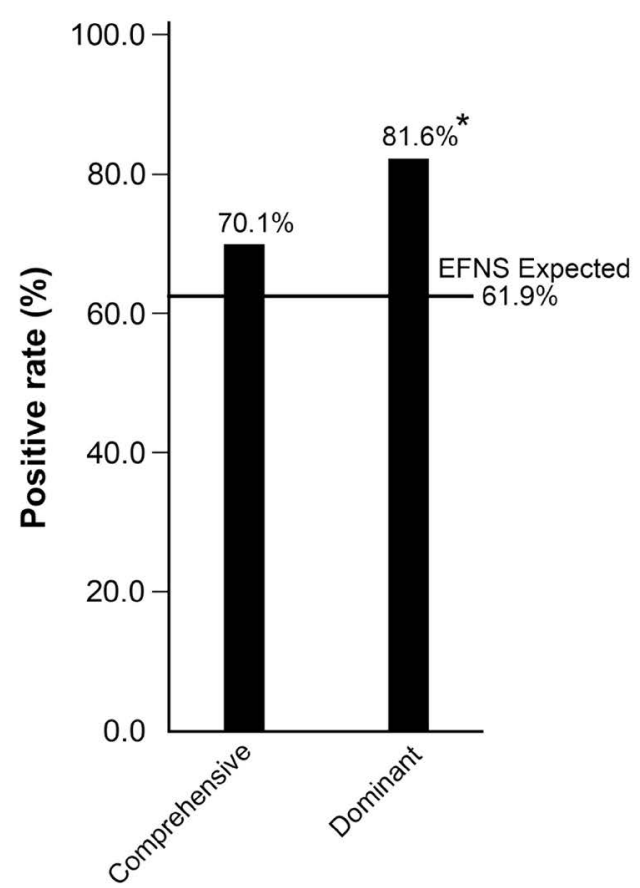

B

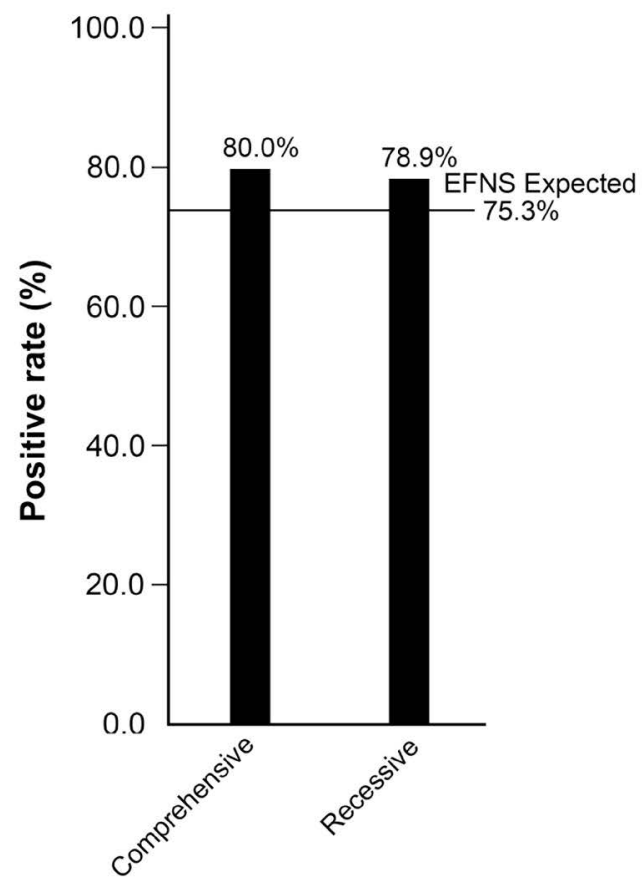

Figure 2. The frequencies of positive results for the (A) dominant loci (SCA1, SCA2, SCA3, SCA6, SCA7, and SCA17) and (B) recessive locus (FRDA) as compared to the observed frequencies published by the European Federation of Neurological Societies (EFNS) in 2014 (horizontal lines) [7]. The frequencies of positive results in the comprehensive and AR profiles were similar to the EFNS data. The frequency of positive results for the AD profile was significantly $(\mathrm{P}<0.001)$ higher compared to the EFNS data.

This study largely supports the EFNS recommendations for genetic testing for ataxia, except for the exclusion of SCA10. The inclusion of SCA8 testing remains controversial due to incomplete penetrance and the finding of co-occurrence with other ataxia gene mutations. Multigene profiles for pathogenic variants in known ataxia genes are especially useful when the inheritance pattern is known.

\section{References}

1. Jayadev S, Bird TD (2013) Hereditary ataxias: overview. Genet Med 15: 673-683. [Crossref]

2. Durr A (2010) Autosomal dominant cerebellar ataxias: polyglutamine expansions and beyond. Lancet Neurol 9: 885-894. [Crossref]

3. Joo BE, Lee CN, Park KW (2012) Prevalence rate and functional status of cerebellar ataxia in Korea. Cerebellum 11: 733-738. [Crossref]

4. Coutinho P, Ruano L, Loureiro JL, Cruz VT, Barros J, et al. (2013) Hereditary ataxia and spastic paraplegia in Portugal: a population-based prevalence study. JAMA Neurol 70: 746-755. [Crossref]
5. Koht J, Tallaksen CM (2007) Cerebellar ataxia in the eastern and southern parts of Norway. Acta Neurol Scand Suppl 187: 76-79. [Crossref]

6. Gasser T, Finsterer J, Baets J, Van Broeckhoven C, Di Donato S, et al. (2010) EFNS guidelines on the molecular diagnosis of ataxias and spastic paraplegias. Eur $J$ Neurol 17: 179-188. [Crossref]

7. van de Warrenburg BP, van Gaalen J, Boesch S, Burgunder JM, Dürr A, et al. (2014) EFNS/ENS Consensus on the diagnosis and management of chronic ataxias in adulthood. Eur J Neurol 21: 552-562. [Crossref]

8. Paulson HL (2009) The spinocerebellar ataxias. J Neuroophthalmol 29: 227-237. [Crossref]

9. Bushara K, Bower M, Liu J, Karen N McFarland, Ivette Landrian, et al. (2013) Expansion of the Spinocerebellar ataxia type 10 (SCA10) repeat in a patient with Sioux Native American ancestry. PLoS One 8: e81342. [Crossref]

10. Zeman A, Stone J, Porteous M, Burns E, Barron L, et al. (2004) Spinocerebellar ataxi type 8 in Scotland: genetic and clinical features in seven unrelated cases and a review of published reports. J Neurol Neurosurg Psychiatry 75: 459-465. [Crossref]

Copyright: (C2017 Wang Z. This is an open-access article distributed under the terms of the Creative Commons Attribution License, which permits unrestricted use, distribution, and reproduction in any medium, provided the original author and source are credited. 